\title{
Maximizing Credibility-based Network Utility via Power Allocation
}

\author{
Ertugrul Necdet Ciftcioglu and Aylin Yener \\ Dept. of Electrical Engineering, The Pennsylvania State University, University Park, PA 16802 \\ Email: enc118@psu.edu, yener@ee.psu.edu
}

\begin{abstract}
We consider Quality-of-Information (QoI)-based resource allocation in a scenario where multiple reporter nodes send information on an event of interest to a sink node. We express credibility of each reporter, an attribute of quality of information, as a function of both the proximity to the event and the type of information gathered. While a reporter close to the event using an advanced format as video may have high credibility, it might also need excessive network resources to transmit the information if it is far from the sink. To address this trade-off, we consider a network utility which depends on both information credibility and timeliness, and yields the timely and credible operational information content capacity (OICC) of this setting. We consider a network with a fixed transmission cost budget per event. Optimal power allocation among reporters is found that maximizes the utility. The constraint on network cost budget results in coupling among resources allocated to reporters. We demonstrate that power allocated to each reporter balances contrasting parameters associated with reporter location and type of information used. Overall, this work produces design insight bringing us one step closer to information quality aware wireless networking.
\end{abstract}

Index Terms-Quality of Information, Credibility, Power Allocation, Network Utility Maximization

\section{INTRODUCTION}

Traditionally, wireless network performance has been characterized by Quality of Service (QoS) metrics as delay, packet loss and fairness. The increasing diversity of applications has called for alternative attributes to characterize the means by which information delivered by the network is useful in decision making. To that end, Quality of Information (QoI), where information is defined by a collection of new alternative Quality descriptors has been of recent interest [1] [2].

These recently considered attributes include accuracy and precision [1] [2], age/freshness and timeliness [1] [2], credibility [2] [3], reliability [1] and provenance [2]. In addition to application-dependent attribute prioritization, QoI-based approach to networking also differs from traditional QoS measures in that typically a piece of information, e.g. a video clip, an audio clip, an image or text message is of interest [2], as opposed to a (long) communication session.

This has lead to recent treatment of wireless networks along with QoI. Initial works which have focused on probability of error for event detection applications [4] [5]. Recently, we have addressed scheduling for optimization of QoI-based utilities for several different network models [6]-[8]. The tradeoff between accuracy and freshness was addressed in [6]. Rate allocation in order to maximize sum QoI utility dependent on accuracy and timeliness has been presented for the multipleaccess channel in [7]. The accuracy-timeliness tradeoff was addressed for a multi-hop relay network with time-varying links in [8], demonstrating the advantages of buffering at relay nodes to exploit opportunistic scheduling.

Methods to characterize credibility and maximize total credibility gained from a network have been presented in [2], [3]. In particular, authors consider a scenario where reporters send information they obtain about an event of interest to a central director. The credibility of information from each user depends on both the type/modality of information and the proximity of the reporter to the event [3].

In this work, we focus on the effect of the credibility attribute on QoI-based utility when information is delivered over wireless channels. To this end, we build on credibilitybased QoI-aware networking in several aspects. First, we take into account distances from reporters to the sink node and its associated effects on transmission in physical media. For instance, while a reporter might be able to have information with high credibility, it might also be far away from the sink node, resulting in increased probability of bit errors for fixed power and bandwidth resources, equivalently increased demand for network resources for a desired transmission quality. Additionally, rather than maximizing credibility alone, we consider a utility function which also depends on the timeliness of information delivered. While a video clip may provide much higher credibility compared to a text message, it would also require delivery of a significantly larger file, which might result in latency at the sink. To address these issues, we consider maximization of a network QoI-utility depending on both credibility and timeliness subject to a fixed budget of transmission energy for the network. We also take into account of file sizes for different modalities for transmission costs, capturing the fact that using modalities resulting in higher credibility requires more transmission resources. We find the optimal allocation of system resources, i.e. the selection of the reporters as well as their optimal allocated powers which maximize the network utility.

The remainder of the paper is organized as follows. In Section II, we present the system model, including details on the credibility model and physical layer. Next, in Section III we present the optimization problem and power allocation solution. After providing numerical results in Section IV, we conclude the paper in Section V. 


\section{SySTEM MODEL}

We consider a scenario where $N$ reporters, indexed by $i \in$ $\{1,2, \ldots, N\}$, are capable of gathering information in different formats, i.e., modalities about an event $E$ (Fig. 1). This model for example, corresponds to surveillance of an area by multiple reporters, or a vehicular network reporting traffic violations, say at an intersection. Reporters send the information to a sink/command node $S$ which makes decisions based on the overall information it receives.

Information reported from each user is characterized by its quality-of-information, represented by a QoI-vector of attributes, e.g., $\mathbf{q}=[$ modality $=$ video, credibility $=$ $C$, timeliness $=15 \mathrm{~s} \ldots]$.

In this work, we focus on the effect of attributes of credibility and timeliness on the decision quality. For credibility of information, we utilize the model in reference [3]. The credibility of information depends on two factors. One is the mode of information, i.e., a clip of video is expected to be more credible than an audio clip, which is generally more credible than a text message. The second factor is based on the logic that a reporter closer to the event is more credible. A reporter who is closer to the event of interest will be able to make observations with higher detail and resolution. Overall, the credibility of reporter $i$ using mode $m$, represented by $c_{i, m}$ is expressed as [3]:

$$
c_{i, m}= \begin{cases}\gamma_{m} d_{e i}^{-\delta_{m}}, & d_{e i}>d_{0} \\ \gamma_{m} d_{0}^{-\delta_{m}}, & d_{e i} \leq d_{0},\end{cases}
$$

where $d_{e i}$ is the distance from the event to reporter $i, \gamma_{m} d_{0}^{-\delta_{m}}$ is the maximum credibility that can be obtained by information of mode $m, \delta_{m}$ characterizes the decay of the credibility of information with distance for mode $m . d_{0}$ is a bound on the distance to prevent division by zero and limit the maximum amount of credibility that can be obtained by a user.

Each modality of information is associated with a file size of $f_{m}$. For instance, a video clip would typically have significantly larger size compared with a text message. Transfer of a larger file size results in two implications; first, it results in longer delivery time, or reduced timeliness. Additionally, it requires more system resources for transmission.

Transmission is performed over wireless channels, which are quasi-static with a path loss factor of $\alpha$. Channels from each reporter to the sink are orthogonal, e.g. a multiple access protocol such as TDMA or OFDMA is in place so that the multiple reporter transmissions do not interfere with each other. We assume that each file is transmitted in packets of length $L$, i.e., mode $m$ requires $\left\lceil\frac{f_{m}}{L}\right\rceil$ packets. The reporters transmit their bits using Differential Binary Phase Shift Keying (DBPSK) modulation, resulting in a bit error rate depending on the power used by reporter $i$ as [9]:

$$
b\left(p_{i}\right)=0.5 e^{-\frac{p_{i} d_{i s}^{-\alpha}}{N_{p}}},
$$

where $p_{i}$ is the power, $d_{i s}$ is the distance from reporter $i$ to sink $s$, and $N_{p}$ is noise power, given by $N_{0} W$ with $N_{0}$ the

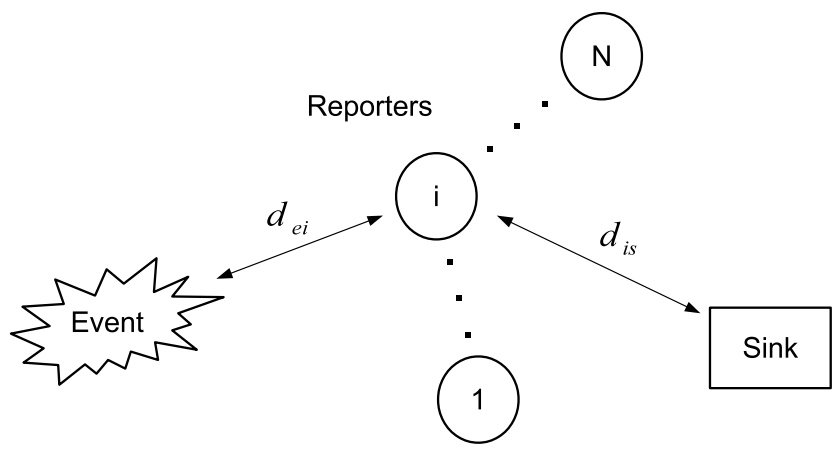

Fig. 1. Network model with $\mathrm{N}$ reporters.

noise spectral density and $W$ is signal bandwidth. The choice of this modulation scheme is strictly for ease of exposition, the basic solutions can be extended to any modulation scheme.

This modulation choice results in a probability of correct reception for a packet of length $L$ that is expressed as [10]:

$$
r\left(p_{i}\right)=\left(1-0.5 e^{-\frac{p_{i} d_{i s}^{-\alpha}}{N_{p}}}\right)^{L} .
$$

The expected number of transmissions to for correct reception of a packet is inversely proportional to $r\left(p_{i}\right)$, see for example [11]. Accordingly, the expected number of transmissions to deliver a file of mode $m$ by reporter $i$ with power $p_{i}$ is given by:

$$
\begin{aligned}
t\left(p_{i}\right) & =\frac{\left\lceil\frac{f_{i, m}}{L}\right\rceil}{r\left(p_{i}\right)} \\
& =\frac{\left\lceil\frac{f_{i, m}}{L}\right\rceil}{\left(1-0.5 e^{-\frac{p_{i} d_{i s}^{-\alpha}}{N_{p}}}\right)^{L}},
\end{aligned}
$$

where $f_{i, m}$ denote the file size for reporter $i$, when it chooses modality $m$. Overall, the QoI-vector of information delivered by reporter $i$ is characterized by $\mathbf{q}_{i}=\{$ modality $=$ $m$, credibility $=c_{i, m}$, timeliness $\left.=t\left(p_{i}\right) \tau_{b}\right\}$, where $\tau_{b}$ denotes bit transmission duration in seconds. We note that this QoI-vector depends on many parameters, as power of reporter $i$, distances from reporter to the event and to the sink, and the modality of operation. Hence, the Quality delivered to the sink from the reporters depends both the initial quality at the reporters and the timeliness of the information. In the following section, we consider the optimization problem maximizing a utility based on the collection of QoI vectors delivered from the network.

\section{QoI-BAsed Utility MAXimization ViA PoWER ALLOCATION}

The maximum QoI-based utility that can be supported by a network has been termed as the Operational Information Content Capacity (OICC) [2] [7]. The QoI utility that can be obtained by a delivered QoI vector is a composite function of several attributes of the vector. In particular, we consider a utility function which reflects the trade-off between the attributes of credibility and timeliness for a QoI-vector $\mathbf{q}$. The 
utility obtained from a QoI-vector considered in this paper should be non-decreasing with credibility and non-increasing with latency. More specifically, for QoI-vector $\mathbf{q}_{i}$ from reporter $i$, we consider the following utility function:

$$
u\left(\mathbf{q}_{i}(c, t)\right)=c g(t) .
$$

While many different $g(t)$ functions can be defined depending on the goal at the sink, in this paper we consider functions of the form:

$$
g(t)=t^{-\beta}
$$

with $\beta \leq 1$ characterizing the extent of the latency penalty. We mainly consider $\beta<1$ in order to soften the penalty due to latency since many applications are relatively tolerant to delay up to critical deadlines. We also note that the case with $\beta=1$ reflects the rate of delivering credibility per unit time from a reporter.

We assume that total network utility is the sum of utilities obtained from all of the reporters. The aim of the network is to maximize this total utility. The utility of a reporter increases with power since expected latency is reduced. However, transmission power in a practical wireless network is a scarce resource. Moreover, the transmission costs increase with larger file sizes. In this sequel, we assume that the network has a total transmission cost budget of $P$. In other words, we consider the following constraint on powers $p_{1}, \ldots, p_{N}$ allocated to reporters:

$$
\sum_{i=1}^{N} h_{m}\left(p_{i}\right) \leq P,
$$

where $h_{m}(p)$ is a relationship for the total cost of sending a file with modality $m$. With the natural choice of minimum total energy spent on transmission, i.e., $h_{m}\left(p_{i}\right)=f_{i, m} \tau_{b} p_{i}$, we have the following constraint

$$
\sum_{i=1}^{N} f_{i, m} \tau_{b} p_{i} \leq P
$$

Accordingly, we are interested in power allocation among reporters to maximize total QoI-based network utility:

$$
\begin{aligned}
\max _{p_{1}, \ldots, p_{N}} & \sum_{i=1}^{N} c_{i, m}\left(\frac{L\left(1-0.5 e^{-\frac{p_{i} d_{i s}^{-\alpha}}{N_{p}}}\right)^{L}}{f_{i, m} \tau_{b}}\right)^{\beta} \\
\text { s.t. } & \sum_{i=1}^{N} f_{i, m} \tau_{b} p_{i} \leq P, \\
& p_{i} \geq 0, \forall i,
\end{aligned}
$$

where we have used $\left\lceil\frac{f_{i, m}}{L}\right\rceil \approx \frac{f_{i, m}}{L}$ for $f_{i, m} \gg L$. This is a constrained optimization problem. The objective function is quasiconcave in $p_{i}$ for general $\beta, L$, and the constraints are linear. Hence, we can solve the problem using Lagrangian methods [12]. The Lagrangian is

$$
\begin{aligned}
J\left(p_{1}, \ldots, p_{N}\right)= & -\sum_{i=1}^{N} c_{i, m}\left(L \frac{\left(1-0.5 e^{-\frac{p_{i} d_{i s}^{-\alpha}}{N_{p}}}\right)^{L}}{f_{i, m} \tau_{b}}\right)^{\beta} \\
& +\lambda\left(\sum_{i=1}^{k} f_{i, m} \tau_{b} p_{i}-P\right)+\sum_{i=1}^{N} \mu_{i}\left(0-p_{i}\right) \\
= & -\sum_{i=1}^{N} c_{i, m}\left(L \frac{\left(1-0.5 e^{-\frac{p_{i} d_{i s}^{-\alpha}}{N_{p}}}\right)^{L}}{f_{i, m} \tau_{b}}\right)^{\beta} \\
& +\lambda\left(\sum_{i=1}^{k} f_{i, m} \tau_{b} p_{i}-P\right)-\sum_{i=1}^{k} \mu_{i} p_{i} .
\end{aligned}
$$

Karush-Kuhn-Tucker (KKT) conditions dictate that

$$
\begin{aligned}
\frac{\partial J}{\partial p_{i}}= & -c_{i, m} L^{\beta} \frac{\left(1-0.5 e^{-\frac{p_{i} d_{i s}^{-\alpha}}{N_{p}}}\right)^{\beta L-1}}{\left(f_{i, m} \tau_{b}\right)^{\beta}} \beta L\left(0.5 \frac{d_{i s}^{-\alpha}}{N_{p}} e^{-\frac{p_{i} d_{i s}^{-\alpha}}{N_{p}}}\right) \\
& +f_{i, m} \tau_{b} \lambda-\mu_{i}=0, \quad \forall i .
\end{aligned}
$$

Hence

$\lambda=\frac{c_{i, m}}{f_{i, m} \tau_{b}} \frac{L^{\beta}\left(1-0.5 e^{-\frac{p_{i} d_{i s}^{-\alpha}}{N_{p}}}\right)^{\beta L-1}}{\left(f_{i, m} \tau_{b}\right)^{\beta}} \beta L\left(0.5 \frac{d_{i s}^{-\alpha}}{N_{p}} e^{-\frac{p_{i} d_{i s}^{-\alpha}}{N_{p}}}\right)+\mu_{i}$,

and using Complementary Slackness Conditions (CSC), the first possibility is that

$$
p_{i}>0 \Rightarrow \mu_{i}=0,
$$

implying that we have

$$
\lambda=\frac{c_{i, m}}{f_{i, m} \tau_{b}} \frac{L^{\beta}\left(1-0.5 e^{-\frac{p_{i} d_{i s}^{-\alpha}}{N_{p}}}\right)^{\beta L-1}}{\left(f_{i, m} \tau_{b}\right)^{\beta}} \beta L\left(0.5 \frac{d_{i s}^{-\alpha}}{N_{p}} e^{-\frac{p_{i} d_{i s}^{-\alpha}}{N_{p}}}\right) .
$$

Let $\tilde{p}_{i}(\lambda)$ denote the power allocation satisfying (18) for a given $\lambda$. The other alternative from CSC is

$$
p_{i}=0 \Rightarrow \mu_{i} \geq 0,
$$

which implies that

$$
\mu_{i}=f_{i, m} \tau_{b} \lambda-c_{i, m} \frac{L^{\beta}(1-0.5)^{\beta L-1}}{\left(f_{i, m} \tau_{b}\right)^{\beta}} \beta L\left(0.5 \frac{d_{i s}^{-\alpha}}{N_{p}}\right) \geq 0,
$$

meaning

$$
\lambda>\frac{c_{i, m}}{f_{i, m} \tau_{b}} \frac{(0.5)^{\beta L-1}}{\left(f_{i, m} \tau_{b}\right)^{\beta}} \beta L^{\beta+1}\left(0.5 \frac{d_{i s}^{-\alpha}}{N_{p}}\right),
$$

i.e.,

$$
\lambda>\frac{c_{i, m} \beta L^{\beta+1} d_{i s}^{-\alpha}(0.5)^{\beta L}}{\left(f_{i, m} \tau_{b}\right)^{1+\beta} N_{p}} .
$$


To summarize, the solution for reporter $i$ is

$$
p_{i}= \begin{cases}0 & \text { if } \frac{c_{i, m} \beta L^{1+\beta} d_{i s}^{-\alpha}(0.5)^{\beta L}}{\left(f_{i, m} \tau_{b}\right)^{1+\beta} N_{p}}<\lambda \\ \tilde{p}_{i}(\lambda) & \text { if } \frac{c_{i, m} \beta L^{1+\beta} d_{i s}^{-\alpha}(0.5)^{\beta L}}{\left(f_{i, m} \tau_{b}\right)^{1+\beta} N_{p}} \geq \lambda,\end{cases}
$$

where $\lambda$ is a function of the total cost budget, and can be found using the total cost constraint, i.e.,

$$
\sum_{i=1}^{N} f_{i, m} \tau_{b} \tilde{p}_{i}(\lambda) \mathbf{1}_{\left\{\frac{c_{i, m} \beta L^{1+\beta} d_{i s}^{-\alpha}(0.5)^{\beta L}}{\left(f_{i, m^{\tau}}\right)^{1+\beta} N_{p}}-\lambda\right\}}=P
$$

where

$$
\mathbf{1}_{\{x\}}= \begin{cases}1 & \text { if } x>0 \\ 0 & \text { if } x \leq 0\end{cases}
$$

denotes the unit step function.

Remark 1: Observe that the solution dictates that the selection of the optimal set of reporters as well as their power levels. A reporter with a 0 power level is one that will simply not report.

While the closed-form solution and associated tradeoffs between different parameters are not directly apparent from (23), for the case $\beta L=1$, we are able to state the following result:

Theorem 1: For $\beta L=1$, the optimal power allocation $p_{i}^{*}$ for reporter $i$ is given by follows:

$$
p_{i}^{*}= \begin{cases}0 & \text { if } \frac{c_{i, m} L^{\beta} d_{i s}^{-\alpha}}{2\left(f_{i, m} \tau_{b}\right)^{1+\beta} N_{p}}<\lambda \\ d_{i s}^{\alpha} N_{p} \log \left(\frac{c_{i, m} L^{\beta} d_{i s}^{-\alpha}}{2 \lambda\left(f_{i, m} \tau_{b}\right)^{1+\beta} N_{p}}\right) & \text { if } \frac{c_{i, m} L^{\beta} d_{i s}^{\alpha}}{2\left(f_{i, m} \tau_{b}\right)^{1+\beta} N_{p}} \geq \lambda\end{cases}
$$

where $\lambda$ satisfies

$$
\sum_{i=1}^{N} f_{i, m} \tau_{b} d_{i s}^{\alpha} N_{p} \log \left(\frac{0.5 c_{i, m} L^{\beta} d_{i s}^{-\alpha}}{\lambda\left(f_{i, m} \tau_{b}\right)^{1+\beta} N_{p}}\right) 1_{\left\{\frac{0.5 c_{i, m} L^{\beta} d_{i s}^{-\alpha}}{\left(f_{i, m} \tau_{b}\right)^{1+\beta} N_{p}}-\lambda\right\}}
$$

and $\mathbf{1}_{\{x\}}$, defined by (25) denotes the unit step function.

Proof: With $\beta L=1$, the first CSC (17) simplifies into

$$
p_{i}>0 \Rightarrow \mu_{i}=0 \text {, }
$$

leading to

$$
\lambda=c_{i, m} \frac{L^{\beta}}{\left(f_{i, m} \tau_{b}\right)^{1+\beta}}\left(0.5 \frac{d_{i s}^{-\alpha}}{N_{p}} e^{-\frac{p_{i} d_{i s}^{-\alpha}}{N_{p}}}\right),
$$

resulting in

$$
p_{i}=d_{i s}^{\alpha} N_{p} \log \left(\frac{c_{i, m} L^{\beta} d_{i s}^{-\alpha}}{2 \lambda\left(f_{i, m} \tau_{b}\right)^{1+\beta} N_{p}}\right) .
$$

The second CSC (20) results in

$$
p_{i}=0 \Rightarrow \mu_{i} \geq 0,
$$

which implies

$$
\mu_{i}=f_{i, m} \tau_{b} \lambda-c_{i, m} \frac{L^{\beta}}{\left(f_{i, m} \tau_{b}\right)^{\beta}}\left(0.5 \frac{d_{i s}^{-\alpha}}{N_{p}}\right)>0,
$$

equivalent to

$$
\lambda>\frac{c_{i, m} L^{\beta} d_{i s}^{-\alpha}}{2\left(f_{i, m} \tau_{b}\right)^{1+\beta} N_{p}} .
$$

Accordingly, the solution is given by

$$
p_{i}= \begin{cases}0 & \text { if } \frac{c_{i, m} L^{\beta} d_{i s}^{-\alpha}}{2\left(f_{i, m} \tau_{b}\right)^{1+\beta} N_{p}}<\lambda \\ d_{i s}^{\alpha} N_{p} \log \left(\frac{c_{i, m} L^{\beta} d_{i s}^{-\alpha}}{2 \lambda\left(f_{i, m} \tau_{b}\right)^{1+\beta} N_{p}}\right) & \text { if } \frac{c_{i, m} L^{\beta} d_{i s}^{-\alpha}}{2\left(f_{i, m} \tau_{b}\right)^{1+\beta} N_{p}} \geq \lambda\end{cases}
$$

where $\lambda$ depends on $P$ and can be found through

$$
\sum_{i=1}^{N} f_{i, m} \tau_{b} d_{i s}^{\alpha} N_{p} \log \left(\frac{0.5 c_{i, m} L^{\beta} d_{i s}^{-\alpha}}{\lambda\left(f_{i, m} \tau_{b}\right)^{1+\beta} N_{p}}\right) \mathbf{1}_{\left\{\frac{0.5 c_{i, m} L^{\beta} d_{i s}^{-\alpha}}{\left(f_{i, m} \tau_{b}\right)^{1+\beta} N_{p}}-\lambda\right\}}=P,
$$

with $\mathbf{1}_{\{x\}}$ is again defined by (25) denotes the unit step function.

Remark 2: From both (23) and (33), it can be observed that power is likely to be allocated to a reporter if its credibility is high, it is closer to the sink, and its file size is small. These factors may severely conflict, since if a reporter is closer to the event to increase credibility, it might be further away from the sink. Likewise, if a reporter uses a mode with higher $\gamma_{m}$, such a decision is also likely to result in a larger file size. The overall optimum solution requires an balances all of these tradeoffs.

\section{Numerical Results}

In this section, we present simulation results supporting our analytical findings. First, we consider a network with 25 reporters. Each node, including the event and sink is uniformly distributed in a two-dimensional region of $1 \mathrm{~km} \times 1 \mathrm{~km}$. We average over 100 network realizations. Each reporter randomly selects one of three modalities of information with equal probability, where modalities have the file sizes $\left[\begin{array}{lll}f_{1} & f_{2} & f_{3}\end{array}\right]=\left[\begin{array}{lll}10^{7} & 5 \times 10^{5} & 10^{4}\end{array}\right]$ bits, and credibility parameters $\left[\begin{array}{lll}\gamma_{1} & \gamma_{2} & \gamma_{3}\end{array}\right]=\left[\begin{array}{lll}0.9 & 0.6 & 0.4\end{array}\right]$, and $\left[\begin{array}{lll}\delta_{1} & \delta_{2} & \delta_{3}\end{array}\right]=$ $\left[\begin{array}{lll}0.4 & 1.2 & 2\end{array}\right] . N_{0}=4 \times 10^{-21}$ Watts $/ H z$ with $W=$ $100 \mathrm{KHz}, \tau_{b}$ assumed to be equal to $\frac{2}{W} \mathrm{sec}, d_{0}=1 \mathrm{~m}$, $L=8$ and $\beta=0.125$ denotes the timeliness emphasis parameter. We consider an environment with path loss factor of 4 . The total cost budget $P$ is varied, and we observe the resulting QoI utility. For comparison purposes, we compare the optimal power allocation algorithm (OPA) with a naive algorithm, (UA), which allocates equal energy expenditure for each reporter.

As observed in Fig. 2, we observe that (OPA) significantly outperforms the credibility-blind algorithm when the cost budget is not very large. When the total cost budget is very large, the cost constraint is not limiting in the sense that the utility saturates and even without proper allocation among reporters, power is sufficient to provide low bit error rates and improved timeliness.

We also present the average percentage of activation for reporters using each modality, in Fig. 3. We observe that re- 


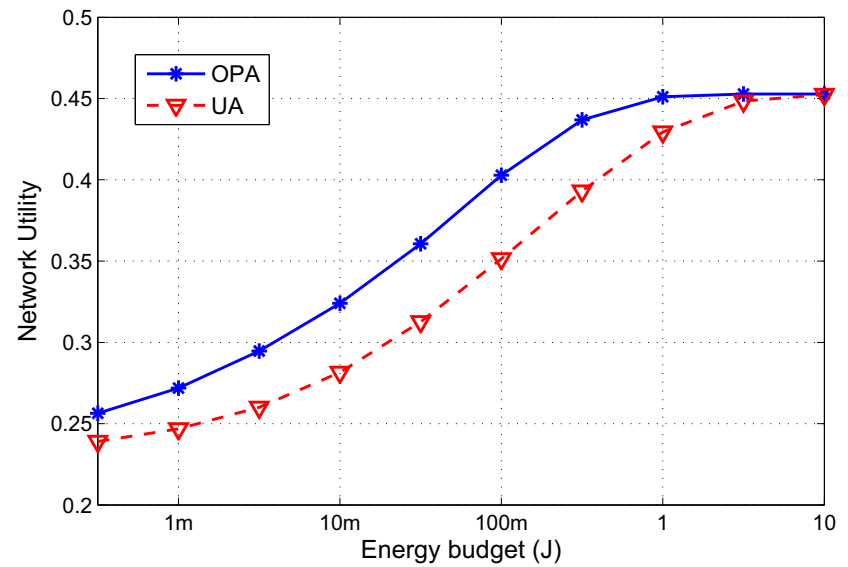

Fig. 2. QoI Utility, Varying cost Budget.

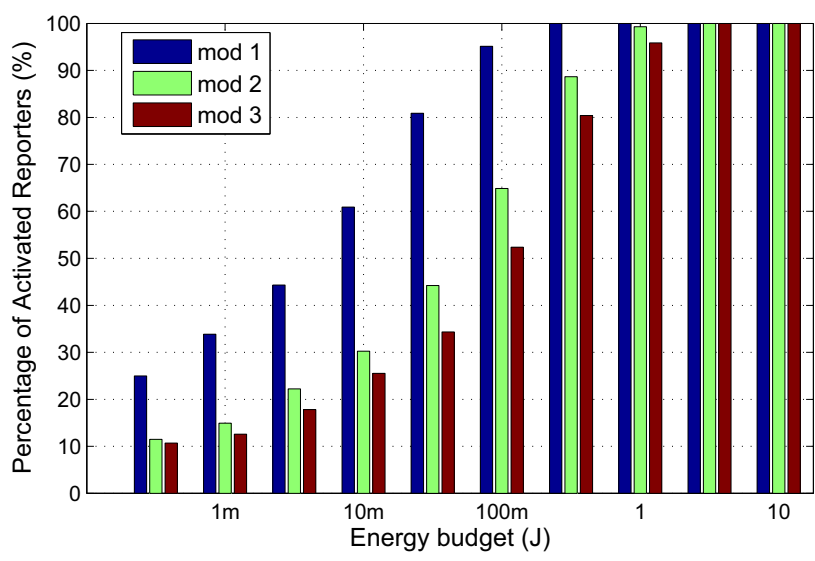

Fig. 3. Percentage of activation for reporters using different modalities.

porters with high credibility tend to be activated in accordance with (33). While the difference is prominent for low cost budgets, with high cost budgets due to abundant resources all reporters are activated.

Next, to demonstrate the power allocation as a function of modality and distances more explicitly, we focus on a two-user scenario with first user using a modality with high credibility (e.g. video) and the second user a modality with modest credibility. Keeping the locations of the first reporter, the event and the sink fixed, we gradually vary the location of the second reporter in one dimension (horizontally), which varies its distance between the event and the sink. The cost budget $P$ is 0.05 Joules. We observe that optimal power allocation is greatly dependent on reporter locations. As shown in Fig. 4-5, power is not allocated to reporter 2 when it is too far away from the event, which reduces the credibility it can provide.

\section{CONCLUSIONS}

In this work, we have considered optimal power allocation for QoI-aware network utility maximization in a scenario where multiple nodes report to a sink on an event of interest. QoI utility from each reporter depends on the QoI attributes credibility and timeliness of information delivered to the sink

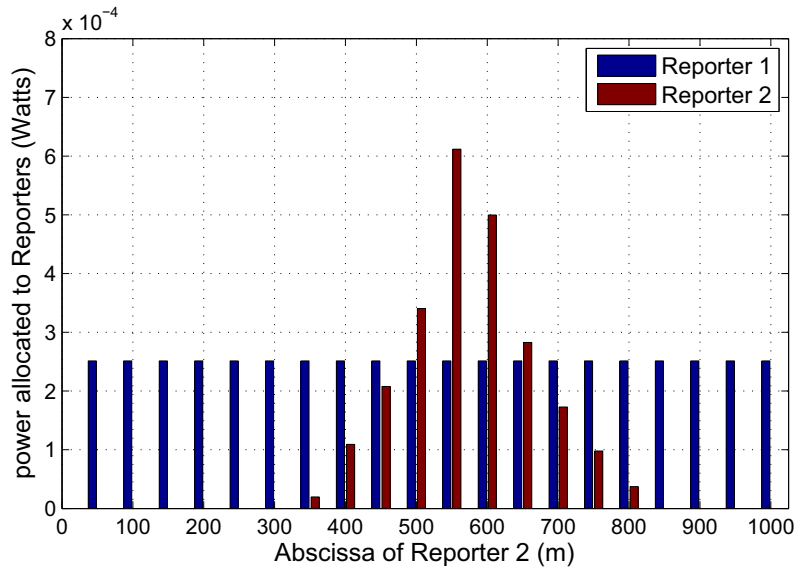

Fig. 4. Optimal power allocation, $p_{i}$ for reporter i.

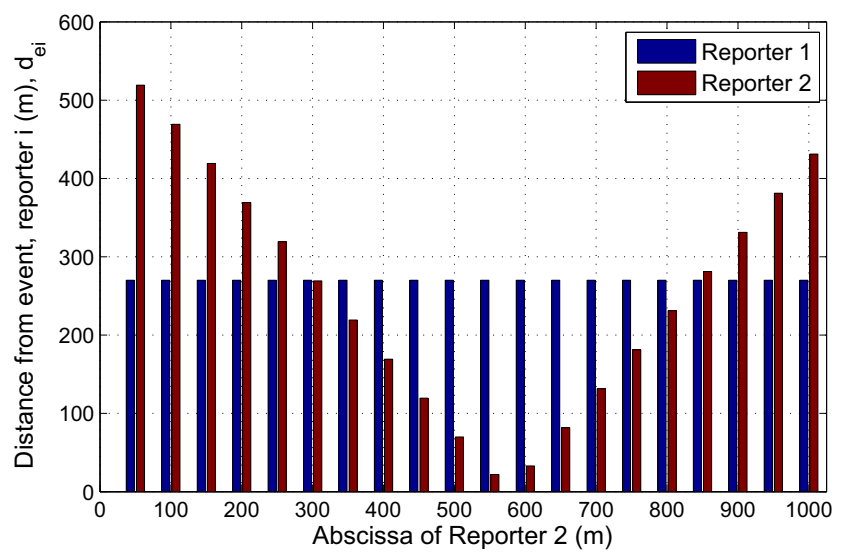

Fig. 5. Distance from event for reporter 2.

by the reporter. We take into account the impact of using different modalities of information in the credibility obtained, as well as transmission through latency and costs. While the effects of reporter location and modality of information might have opposing effects on credibility and timeliness attributes separately, our power allocation solution optimally balances these effects to maximize the overall QoI utility depending on multiple attributes.

This paper contributes to QoI-based wireless networking by addressing the combination of new attributes, also taking a cross-layer approach for trading off effects associated with higher-level attributes and lower-layer effects due to transmission. Future work includes integrating modality selection for each reporter developing a joint modality selection-power allocation optimization.

\section{ACKNOWLEDGEMENT}

Research was sponsored by the U.S. Army Research Laboratory under the Network Science Collaborative Technology Alliance, Agreement Number W911NF-09-2-0053. The views and conclusions contained in this document are those of the author(s) and should not be interpreted as representing the official policies, either expressed or implied, of the U.S. Army 
Research Laboratory or the U.S. Government. The U.S. Government is authorized to reproduce and distribute reprints for Government purposes notwithstanding any copyright notation hereon.

\section{REFERENCES}

[1] S. Zahedi and C. Bisdikian. A Framework for QoI-inspired analysis for sensor network deployment planning. In International Workshop on Performance Control in Wireless Sensor Networks, PWSN, Austin, TX, October 2007.

[2] A. Bar-Noy, G. Cirincione, R. Govindan, S. Krishnamurthy, T. F. LaPorta, P. Mohapatra, M. Neely, and A. Yener. Quality-of-Information Aware Networking for Tactical Military Networks. In Third International Workshop on Information Quality and Quality of Service for Pervasive Computing, in conjunction with IEEE Percom 2011, Seattle, WA, March 2011.

[3] B. Liu, P. Terlecky, A. Bar-Noy, R. Govindan, and M. J. Neely. Optimizing Information Credibility in Social Swarming Applications. In IEEE INFOCOM 2011 mini-conference, Shanghai, China, April 2011.

[4] Z. M. Charbiwala, S. Zahedi Y. Kim, Y.H. Cho, and M. B. Srivastava. Toward Quality of Information Aware Rate Control for Sensor Networks. In Fourth International Workshop on Feedback Control Implemenation and Design in Computing Systems and Networks, San Francisco, CA, April 2009.

[5] C. Bisdikian. On Sensor Sampling and Quality of Information: A Starting Point. In IEEE Conference on Pervasive Computing and Communication Workshops, White Plains, NY, March 2007.

[6] R. Urgaonkar, E. N. Ciftcioglu, A. Yener, and M. J. Neely. Quality of Information Aware Scheduling in Task Processing Networks. In Proc. The 7th International Workshop on Resource Allocation and Cooperation in Wireless Networks (RAWNET), in conjunction with IEEE WiOpt 2011, Princeton, NJ, May 2011.

[7] E. N. Ciftcioglu, A. Yener, R. Govindan, and K. Psounis. Operational Information Content Sum Capacity: Formulation and Examples. In Proc. 14th conference on Information Fusion, Chicago, IL, July 2011.

[8] E. N. Ciftcioglu and A. Yener. Quality-of-Information Aware Transmission Policies with Time-Varying Links. In Proc. MILCOM 2011, Baltimore, MD, November 2011.

[9] S. Haykin and M. Moher. Modern Wireless Communication. Prentice Hall, 2005.

[10] J. G. Proakis and M. Salehi. Digital Communication, 4th ed. McGrawHill, 2000.

[11] A. Leon-Garcia and I. Widjaja. Communication Networks : Fundamental Concepts and Key Architectures, 2nd ed. McGraw-Hill, 2004.

[12] M. S. Bazaraa, H. D. Sherali, and C. M. Shetty. Nonlinear programming, 3rd ed. John Wiley and Sons, Hoboken, NJ, 2006. 